\section{Check for updates}

Cite this: Lab Chip, 2020, 20, 434

DOI: $10.1039 / c 9 l c 90130 b$

rsc.li/loc

\title{
Correction: Fluid displacement during droplet formation at microfluidic flow-focusing junctions
}

\author{
Haishui Huang ${ }^{\mathrm{ab}}$ and Xiaoming $\mathrm{He}^{\star \mathrm{bcd}}$
}

Correction for 'Fluid displacement during droplet formation at microfluidic flow-focusing junctions' by Haishui Huang et al., Lab Chip, 2015, 15, 4197-4205.

It was brought to our attention that the diffusion coefficient in the original ref. 44 is not the diffusion coefficient of amaranth dye in water, as stated in the original article, but the diffusion coefficient of water in amaranth. The authors regret this inappropriate reference and hope that their explanation given below is helpful to future readers of this paper.

After further studying the literature, it seems that the diffusion coefficients of amaranth dye in water and aqueous carboxymethyl cellulose solution in our study are still unavailable. However, the diffusion coefficients of amaranth dye in agar gel of various concentrations were reported (Fig. 1), ${ }^{1}$ and they follow a linear relationship with the agar concentration $\left(R^{2}=0.9906\right)$. Therefore, we may extrapolate the diffusion coefficient of amaranth dye in water (i.e., when the agar concentration is 0 ) to be $6.377 \times 10^{-10} \mathrm{~m}^{2} \mathrm{~s}^{-1}$. Because the aqueous carboxymethyl cellulose solution is much more viscous than water, the diffusion coefficient of amaranth dye in carboxymethyl cellulose solution should be much smaller than $6.377 \times 10^{-10} \mathrm{~m}^{2} \mathrm{~s}^{-1}$. As a result, the Peclet Number will be at least 485, which is still much larger than 1. So, our conclusion in the paper "the transport of the dye in the core fluid is dominated by advection rather than diffusion" is still valid.

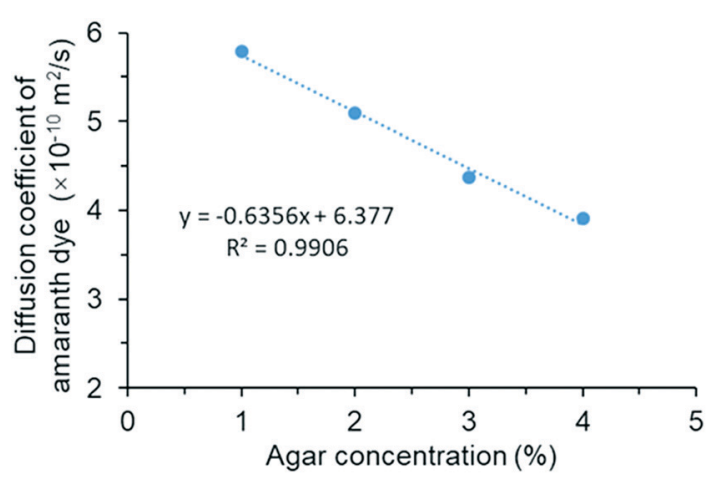

Fig. 1 The relationship between the diffusion coefficient of amaranth dye and agar concentration.

The Royal Society of Chemistry apologises for these errors and any consequent inconvenience to authors and readers.

\section{Notes and references}

1 W.-Y. Lee and Y.-H. Choi, Han'guk Sikp'um Kwahakhoechi, 1991, 23, 217-223.

\footnotetext{
${ }^{a}$ Department of Mechanical and Aerospace Engineering, The Ohio State University, Columbus, Ohio 43210, USA

${ }^{b}$ Department of Biomedical Engineering, The Ohio State University, 308 BRT, 473 W. 12th Avenue, Columbus, Ohio 43210, USA. E-mail: shawnhe@umd.edu;

Fax: +1 614292 7301; Tel: Phone: +1 6142478759

${ }^{c}$ Davis Heart and Lung Research Institute, The Ohio State University, Columbus, Ohio 43210, USA

${ }^{d}$ Comprehensive Cancer Center, The Ohio State University, Columbus, Ohio 43210, USA
} 